\title{
Dynamic Electronic Commerce based Novel Management Platform
}

\author{
Jinhai Lu \\ Guangxi Polytechnic (GXP) \\ 271092619@qq.com
}

Keywords: Electronic Commerce; Management Platform; Dynamic System; Theoretical Analysis.

\begin{abstract}
In this paper, we research on dynamic electronic commerce based novel management platform. The majority of logistics information system is a closed and fixed, its strain capacity is not strong, hindered the enterprise development. E-commerce negotiation is an important problem in electronic commerce research, is essential links of e-commerce activities, it is of great practical significance to the development of electronic commerce. For dynamic e-business, take the customer demand as the leading development model, will replace the past from the inside to the outside of that technology development model. In the paper, e discuss the issues in detail.
\end{abstract}

\section{Introduction}

With the emergence of web services technology, the electronic commerce to the evolution of dynamic e-business, dynamic e-business is actually able to dynamically adapt to changing business enterprise strategy of e-business processes and related systems. It reflects the dynamic integration application under the condition of real-time implementation of the concept, reflects the true value of e-commerce, enterprise logistics system is a comprehensive variety of information sources, constantly optimize the decision-making system. The majority of logistics information system is a closed and fixed, its strain capacity is not strong, hindered the enterprise development. And the logistics system for dynamic e-business can make the enterprise to build the dynamic and valuable electronic relations, continuous improvement on existing information application platform and add new application and function of catering to the modern enterprise to commercial interests driven and is not limited to the trend of the technology. E-commerce negotiation can achieve consistency, determines the success or failure of the entire business activities. E-commerce negotiation is an important problem in electronic commerce research, is essential links of e-commerce activities, it is of great practical significance to the development of electronic commerce. E-commerce negotiation is traditional negotiation theory and method was applied to modern electronic business activities, with the aid of computer and network technology, solve the conflict between the two sides in the commercial negotiations, is the negotiations both sides to reach a consistent, complete business activities important way. To negotiate further study will certainly promote the electronic commerce development in the direction of intelligent, scientific and humanistic that is meaningful [1-2].

In the electronic commerce negotiation, negotiation model and the algorithm is the focus of experts and scholars at home and abroad and the key problem. The negotiations both sides according to their own interests in the process of negotiation and the other party to provide information consultation, seeking benefit maximization and make agreement with each other as much as possible. Now, have a lot of negotiation model and algorithm have been proposed, but due to the environment, object and solve the problems of each are not identical, yet it is not common e-commerce negotiation model and algorithm. For dynamic e-business, take the customer demand as the leading development model, will replace the past from the inside to the outside of that technology development model, and the application requires a high degree of customization, and can be instant quickly assembled into new applications. So, the best solution is to use the Web service technology, the various applications and encapsulation, combination and integration of information system in order to achieve the goal of dynamic e-business [3].

This technique simplifies the system integration, can be quick and easy to join and transaction processing systems, without considering the application environment is what kind of platform on the 
implementation of systems and equipment. Compared with other systems integration technology, it is not a model or architecture around a certain type of systems technology, but for the general application integration and development of the innovation of technology to the problem. The core business functions encapsulated with Web services into components, these business functions can be easily shared between enterprises. Based on Web service components are deployed to the network at once, all connected to the network application can be invoked at any time and integrate these Web services. Through the adoption of Web services, can significantly reduce the cost of the development, deployment and integration, developers do not need to interact with a variety of platforms and programming languages, only need to have the standard protocol for Web services [4-5].

To optimize the current pattern, in this paper, we research on dynamic electronic commerce based novel management platform. The development of modern electronic commerce needs buyers and sellers, Banks, government agencies, certification bodies, institutions such as mutual cooperation and coordination with the logistics center. Complete meaning of e-commerce is not a traditional version of the physical world business activities and it needs the electronic certification, online electronic payments, network security, electronic signature and other technical support, also need to the national development of the construction of the electronic commerce system security. Therefore must be the government departments, the authentication institutions, Banks, network communication, enterprises and other departments jointly promote and participate in, to ensure the whole stability and reliability of the electronic business activities and to develop quickly and well.

\section{The Proposed Methodology and Designed System}

The General Introduction of Electronic Commerce. Information is the development of the world economy after industrialization and a revolution, is the world's economic and social development trend, is any enterprise is unable to avoid. E-commerce as an important part of national economy information and enterprise information, in all walks of life will have far-reaching influence. At present, the development of e-commerce is represented by the United States to the global scope rapid expansion. From the perspective of the development trend of technology and economy, after several years of global business information, transfer mainly through the Internet, the network will become the nervous system of the commercial society in the future and e-commerce will become the mainstream of social economic and trade pattern in the future.

With the development of economy and technology, electronic commerce as a new business form, will change the enterprise production, management, management mode which will largely promote the development of enterprise information. Electronic commerce's biggest feature is crossing the barriers of time and space in traditional sales model, direct implementation the use of network communication for business communication between the seller and the buyer, it not only fast and convenient, and can reduce production costs and trade costs, prompted enterprise production and business operation mode and the transformation of the economic structure, the modern economic activity have a significant impact.

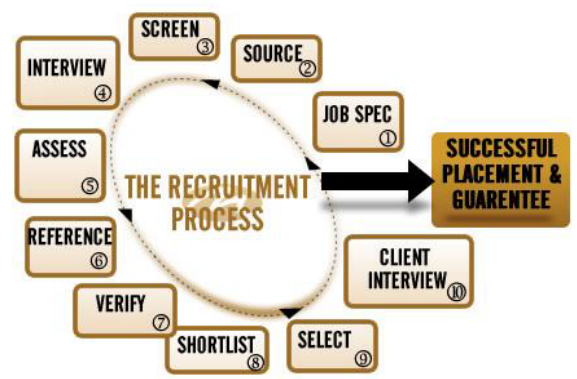

Electronic commerce is the key of enterprise development, e-commerce in helping export-oriented enterprises to adjust market, nature inspired processing enterprise development design brand innovation which will guide the small and the medium-sized enterprise implement information management has an irreplaceable role in many aspects. With the development of network 
technology and computer technology, more and more enterprises to develop e-commerce as an important part of enterprise development strategy, to improve the core competitiveness of the enterprise will have important meaning and function.

The Principles of Dynamic Electronic Commerce. Traditional production-oriented enterprises to realize the process of electronic commerce is a gradual process, is not necessarily one pace reaches the designated position. Generally speaking, domestic production enterprises to develop e-commerce, is formed in the enterprise internal network, realize the enterprise organization and management, production management, financial management, information management, and other internal business electronically on the basis of conduct. Therefore, the first step is to build your own website, introduce their products and services: the second step is to allow visitors to the site to interact with the enterprise, through real-time information communication, Internet chat rooms, bulletin boards and other forms to realize the exchange of information; At the same time, according to their own product attributes, and their own advantages, according to the demand of the market, design to create your own network brand, has a clear and clear market positioning, to carry out the product image promotion, make into the corporate website of netizens to the enterprise and products have a good knowledge and understanding. At present, the production of enterprise electronic commerce's biggest problem is the lack of talent. Enterprise development in the field of electronic commerce requires interdisciplinary inter-disciplinary talent, the talent should not only master the Internet technology, and master of business administration knowledge.

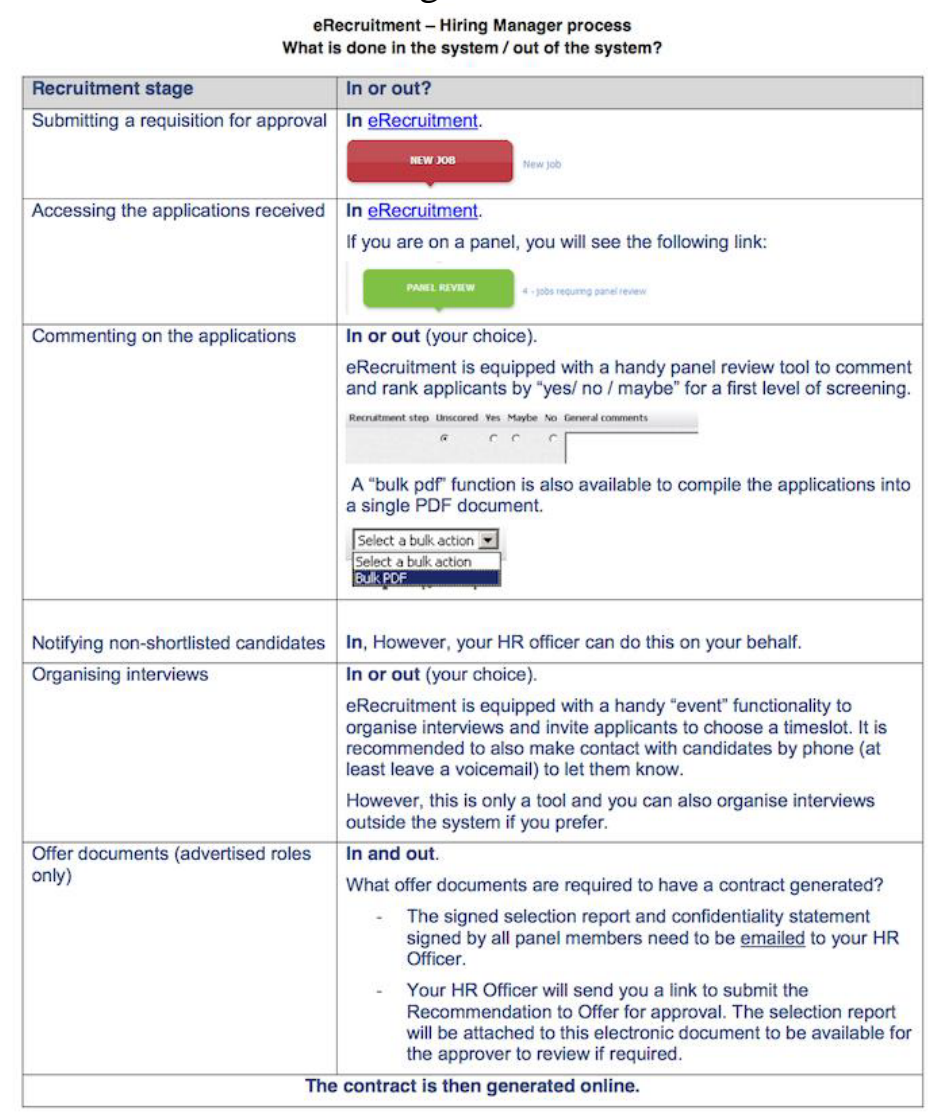

At present, the domestic traditional enterprises are more passive in electronic commerce and more is to online retail as a supplement of the physical channel, or even just a placeholder ornament. For the future of e-commerce positioning, may also just "another leg to walk". Traditional enterprises to develop e-commerce, the purpose is to fully into the network economy era, to take advantage of new e-commerce model to carry out business activities, to provide customers with convenient, fast, high-quality services. Under the wave of global economic integration, build a supply chain system as well as the collaborative e-commerce model is to enhance the competitiveness of enterprises an effective path. Take the concept of unity, like term consolidation method and fusion research is combed for e-commerce platform of supply chain coordinated management, supply chain 
collaborative management mode of e-commerce, e-commerce logistics management and the integration of e-commerce supply chain coordination management.

The Prospect and Summary. The field research in connotation. Or perspective on diversified characteristics, systematic combing is needed to guide the future related research. Trying to build e-commerce supply chain coordination management research framework and divided into supply chain collaborative management, integration of supply chain coordination with e-commerce, supply chain collaborative e-commerce technology three dimensions and information management. Supply chain can be integrated with the e-commerce collaborative research generally divided into e-commerce platform, e-business supply chain collaborative management model of two areas. The face of collaborative e-commerce and the trend of supply chain integration inevitably, scholars mainly study the e-commerce logistics management and supply chain integration management. Healthy sustainable development of the e-commerce market and improve the credit consciousness is the fundamental problem and the credit is the most important. From the point of credit evaluation system, the existing evaluation results are calculated through a variety of model "credit rating", not to establish an index to describe the degree of credit changes, used to describe the evaluation object is more and more faithful or does not keep your words. Dynamically estimate the counterparty, consider the recent and historical evaluation and the combination of trading, more comprehensive response to the credit situation, online transactions to both sides to distinguish bad reputation. And data mining perspective, there has been no scholar in C2C involves huge amounts of data to contribute to the quantitative analysis and data mining, the current empirical research data also many for computer simulation data, the lack of real data test. Mobile terminals and the life of people to contact, on the other hand, personal credit evaluation theory can be applied to mobile business, open up a new research direction of mobile commerce, and improve the system of credit evaluation.

\section{Conclusion and Summary}

In this paper, we research on dynamic electronic commerce based novel management platform. Because anybody in the world with Internet access could potentially see your job posting, you may be inundated with responses, many of them from unqualified candidates. You'll have to take time that you may not really have to wade through each application. To avoid unwanted applications, you'll need to be careful as to how you word your job posting and be as specific as possible about the job duties and the qualifications you're seeking. The face of collaborative e-commerce and the trend of supply chain integration inevitably, scholars mainly study the e-commerce logistics management and supply chain integration management. In the future, more related research will be finalized.

\section{References}

[1] Kim K, Tse E. Dynamic Competition Strategy for Online Knowledge-Sharing Platforms [J]. Social Science Electronic Publishing, 2010, 16(1):41-76.

[2] Ferdous, M. S., \& Poet, R. (2014). Managing dynamic identity federations using security assertion markup language (saml). Journal of Theoretical \& Applied Electronic Commerce Research.

[3] Wu, J. (2014). Study on the design of virtual apparel fitting system and the application of motion capture technology in electronic commerce. Applied Mechanics \& Materials, 519-520.

[4] Resinas, M., Fernández, P., \& Corchuelo, R. (2014). Automatic service agreement negotiators in open commerce environments. International Journal of Electronic Commerce, 14(3), 93-128.

[5] Zhao, X., \& Johnson, M. E. (2010). Managing information access in data-rich enterprises with escalation and incentives. International Journal of Electronic Commerce, 15(1), 79-112. 